\title{
Geopolítica de las tecnologías estratégicas y no estratégicas
}

\section{Geopolítica das tecnologias estratégicas e não estratégicas}

\author{
Daniel Blinder ${ }^{1}$
}

1. Licenciado en Ciencia Política y Doc-
tor en Ciencias Sociales, Universidad de Buenos Aires. Licenciado en Ciencia Política y Doctor por la Facultad de Ciencias Sociales, Universidad de Buenos Aires (UBA). Investigador del Centro de Estudios de Historia de la Ciencia y la Técnica José Babini, Universidad Nacional de San Martín (UNSAM), Provincia de Buenos Aires, Argentina. Profesor de Geopolítica (UBA) y de Tecnología y Relaciones Internacionales (UNSAM) Email: dblinder@unsam.edu.ar ORCID: 0000-0002-9318-7040 (iD)

\section{RESUMEN}

Este artículo se propone estudiar la locación geográfica de la fabricación de las principales compañías tecnológicas A partir de estudios de casos se puede inferir que la producción de las tecnologías estratégicas en la era de la globalización gozan de protección, incentivos, y producción en el país en el cual están radicadas, y por el contrario, aquellas que no son estratégicas se trasladan a otro país con el objeto de optimizar la producción y las ganancias. Los casos a analizar serán las 5 principales compañías de alta tecnología para uso comercial-civil, y para uso de seguridad nacional-militar. La metodología constirá en relevar y analizar la información de las empresas, localizar sus principales plantas de producción, y realizar un análisis descriptivo y explicativo. Los hallazgos del presente trabajo son que las cinco empresas que no consideramos estratégicas producen en serie en China por cuestiones de mercado, y aquellas tecnologías estratégicas tienen sus plantas de fabricación en territorio estadounidense por cuestiones de seguridad nacional y frontera tecnológica.

Palabras Clave: Geopolítica; Tecnología; Producción; China; Estados Unidos

\section{RESUMO}

Este artigo propõe estudar a localização geográfica da fabricação das principais empresas tecnológicas. De estudos de caso, pode-se inferir que a produção das tecnologias estratégicas na era da globalização goza de proteção, incentivos e produção no país em pelo contrário, aqueles que não são estratégicos são transferidos para outro país, de modo a otimizar a produção e os lucros. Os casos a serem analisados serão as 5 maiores empresas de alta tecnologia para uso comercial-civil e para uso de segurança nacional-militar. A metodologia será retransmitir e analisar a informação das empresas, localizar suas principais plantas de produção erealizar uma análise descritiva e explicativa. Os resultados do presente trabalho são que às cinco empresas que não consideramos produtos estratégicos em série na China para questões de mercado e essas tecnologias estratégicas têm suas fábricas nos Estados Unidos para a segurança nacional e fronteira tecnológica.

Palavras Chave: Geopolítica; Tecnologia; Produção; China; Estados Unidos 
Introducción

Las afirmaciones de la filosofía hermética cuyo principal postulado es que tanto es arriba como abajo en el plano físico, y también en el espiritual, parece aplicar para grandes abstracciones y para entender la composición del universo. El macrocosmos y el microcosmos, son parte de un mismo proceso. Así, la división Tierra-Cielo como arriba-abajo también parece relativa si tomamos en cuenta el lugar que ocupamos en el universo. Sin embargo, esta afirmación no aplica para cuestiones de política tecnológica. Un sistema tecnológico como las telecomunicaciones necesita satélites, lanzadores, antenas, cables, pero también teléfonos celulares o computadoras para concretar la comunicación. No parece igual de estratégico desde una perspectiva geopolítica, es decir, con un enfoque de anclaje territorial y de relaciones de poder, fabricar lo que está arriba o lo que está abajo. Ambos son mercados de producción y consumo codiciados, pero unos son estratégicos y otros no lo son. Lo mismo sucede con las tecnologías militares más simples, como las armas de fuego que son producidas en varios países, pero cuando se trata de tecnologías militares complejas como la aeroespacial, estas parecen hacerse localmente.

El presente trabajo tiene por objetivo estudiar la locación de la producción de las principales compañías de tecnologías aeroespaciales y satelitales por un lado, y de las principales compañías de computadoras, Internet y comunicaciones, o relacionadas, por el otro. ¿Por qué elegimos empresas fabricantes de estas últimas y aeroespaciales? Porque ambas son tecnologías de punta para las comunicaciones, ambas están interconectadas en el proceso de la telecomunicaciones, unas se utilizan en mano del usuario, la otra conectando y transmitiendo desde su órbita, una es no estratégica porque es de consumo masivo, la otra si lo es porque requiere ciertos saberes y capacidades, son de uso dual, sensibles, y de propósito general.

En ese sentido se buscará determinar si efectivamente, a partir del estudio de estos casos se puede inferir que la producción de las tecnologías estratégicas en la era de la globalización gozan de protección, incentivos, y producción en el país en el cual están radicadas sus casas matrices, y en cambio, aquellas que no son estratégicas se trasladan a otro país con el objeto de optimizar la producción abaratando costos. Los controles de exportación son un instrumento utilizado por los aquellos Estados que buscan desarrollarse y controlar que su tecnología no sea compartible. Los Estados Unidos, país de cuyas empresas haremos el presente análisis, han hecho uso de esta herramienta en varias ocasiones, a pesar de la libertad de empresa y de investigación académica (CUPITT, 2000). Hay normativa muy específica al respecto, por ejemplo la Directiva de Seguridad Nacional sobre la Política de Transferencia Científica, Técnica o de Ingeniería (National Security Decision Directive 189 - National Policy on Transfer of Scientific, Technical and Engineering Information) o las Normas I.T.A.R. (International Traffic in Arms Regulations, que regulan las exportaciones de componentes y tecnologías sensibles). Así por ejemplo se establece que

"esta directiva establece una política nacional para el control del flujo de la información sobre investigación fundamental de ciencia, tecnología e ingeniería, producida por agencias de financiamiento federal en universidades y laboratorios. Se define como investigación fundamental a [...] la investigación básica y aplicada 
2. Boeing (puesto 73), Lockheed Martin (puesto 152), General Dynamics (puesto 230), Raytheon (puesto 285), Northrop Gruman (puesto 297). Apple (puesto 9), Microsoft (19), Alphabet (puesto 24), IBM (puesto 43), y Amazon (puesto 83). en ciencia e ingeniería, los resultados que son ordinariamente publicados y compartidos con la comunidad científica, la investigación privada y el desarrollo industrial, el diseño, la producción, y la utilización del producto, por cuestiones de propiedad o razones de seguridad nacional” (NSDD 189).

Metodológicamente se relevará y analizará la información, se analizará la política y el mercado de la compañía trabajada, para luego localizar su principal o principales plantas de producción, mediante la selección de un recorte de aquellas compañías que se encuentran posicionadas como principales en el ranking de Forbes. Se descartará toda la lista de empresas por resultar una limitación para el estudio en universo completo, y se tomarán las primeras cinco por corroborar ellas el planteo inicial.

Si efectivamente las 5 estratégicas y las 5 no estratégicas cumplen con las características planteadas, y sólo las que son estratégicas producen en el territorio del país de origen, esto simplemente corrobora el hecho de que éstas por cuestiones de seguridad y soberanía fabrican en dichas locaciones. En cambio, en relación a las no estratégicas es más complejo, y se han tomado a modo de cláusula metodológica -dada la multiplicidad de compañías- sólo las que producen mediante el outsourcing o subcontratación con la gran fabricante taiwanesa Foxconn. El criterio específico son las principales empresas que Forbes denomina en la categoría comunicaciones, Internet o computadoras que fabrican alguna línea de sus productos de marca con Foxconn, lo que nos permite centralizar la mirada en esa compañía que nació en Taiwán, dado que hablamos de unos productos que son masivos, múltiples en sus tipos y modelos, y hay gran cantidad de marcas y países que también lo producen.

Las estratégicas son menos en cantidad, y también tienen líneas de producción civil, pero descartamos esa línea comercial aquí por ser no estratégicas. Por otro lado, hemos optado por la cláusula metodológica de tomar las cinco estratégicas enmarcadas en empresas que se encuentran en el ranking mencionado, que producen tecnología satelital y aeroespacial con fines militares. No hacemos distinción de productos, con el mero objetivo de mirar dónde y no cómo, a partir del por qué.

Se podrá argüir que no representan todo el universo, pero las ciencias sociales, como otras disciplinas, a partir de la selección de una unidad de análisis representativa, tratan de explicar un fenómeno. En todo caso el alcance explicativo del presente artículo es limitado, y será objeto de próximos escritos ampliar esta información en los casos analizados. En China, decenas de empresas importantes como AT \& T, Abbot Laboratories, Adidas, Avon, Black \& Decker, Coca-Cola, Compaq, Dell, DHL, General Electric, General Motors, Nestlé, RCA, y UPS, entre muchas otras. Estas y otras podrían producir en territorio chino o en otro que le otorgue ventajas comparativas. Sin embargo, excede al propósito del presente trabajo.

Este trabajo analizará la geopolítica de las industrias estratégicas a partir del análisis de los casos de las 5 principales compañías ${ }^{2}$ de alta tecnología de acuerdo al ranking que realiza Forbes a partir de ventas, valores de mercado, activos, y ganancias en el año 2017, subdividas en aquello que Forbes clasifica como industrias de Defensa y Aeroespaciales por una parte -que consideramos estratégicas-, y por otra aquellas que son vinculadas a las de tecnología en los rubros comunicaciones, 
internet, computadoras. Así las empresas estratégicas serán la Boeing, Lockheed Martin, General Dynamics, Raytheon, Northrop Gruman, y las no estratégicas a trabajar serán Apple, Microsoft, Alphabet, IBM, y Amazon (FORBES, 2017). Las cinco empresas que no consideramos estratégicas producen en serie en China. Se verán las relaciones de poder en clave geográfica: las estratégicas tienen sus plantas de fabricación en territorio estadounidense.

Tecnologías estratégicas

¿Qué se quiere decir en este artículo con el término tecnología estratégica? Siguiendo a Lawrence Gershwin y Frank Gac (2014, p. 3), "latencia estratégica refiere al inherente potencial de una tecnología de crear cambios militares o económicos en el balance de poder. Este potencial podría permanecer inexplotado e incluso no reconocido, y por lo tanto, latente, hasta que la combinación de factores juntos produzcan poderosas capacidades". En efecto, toda tecnología es usada o puede ser usada para ejercer poder económico o militar. Por lo tanto, definimos que son tecnologías estratégicas aquellas que son de uso dual (LEHMAN, 2014) pero que además tienen un intrínseco valor estratégico por ser económicamente rentables, tecnologías vinculadas a sectores estratégicos de la industria.

El crecimiento y dinamismo creado por estas tecnologías es de naturaleza distinta del motivado por innovaciones incrementales. La máquina a vapor, el motor a combustión, la electricidad, la energía nuclear, las computadoras o la propia tecnología espacial y de telecomunicaciones se enmarcan dentro de esta categoría. Una tecnología así produce en términos de Schumpeter "vendavales de destrucción creativa" e impulsan a los sectores industriales que lideran la economía global, y puede tener un período de desaceleración prolongada en el cual se produce un período de reorganización, rediseño de instituciones y aprendizaje que exigen las complejas transformaciones necesarias para la asimilación de la nueva tecnología por parte de las estructuras productivas de las economías de los países centrales, que son las que lideran e impulsan estos procesos, para luego tener un período de rápido y explosivo crecimiento (HURTADO, et al., 2017; RUTTAN, 2006).

De acuerdo a Chase-Dunn y Reifer (2002) las nuevas industrias, "son importantes como plataforma para el ascenso hegemónico porque producen enormes beneficios indirectos para las economías nacionales en las cuales emergen (...) y porque generan 'rentas tecnológicas"”. El origen de los enormes beneficios que se obtienen debe buscarse en las políticas tecnológicas e industriales que diseñan los países centrales con el objetivo de construir posiciones dominantes a través del desarrollo y control de las tecnologías y por lo tanto conducen, prolongan y orientan los ciclos hegemónicos de las potencias, y las tecnologías que son consideradas de punta que están en la base de estas nuevas industrias. ¿Qué define a un sector industrial como estratégico? La respuesta es que "[...] debe envolver grandes externalidades positivas, grandes economías de escala basadas en el aprender haciendo, e importantes vínculos tanto hacia arriba como hacia abajo” (MICHALSKI, 1991, p. 9). En consecuencia, son dinámicas, tienen 
3. Someramente, un país central es aquel que tiene desarrollo económico y social, y por lo tanto mayor poder político. Sus industrias y tecnologías son avanzadas, y está a la vanguardia de la investigación, desarrollo, innovación e inversión. Por el contrario, un país periférico tiene las características opuestas a los centrales. En cambio, la semiperiferia es periferia pero con ciertos niveles de desarrollo industrial y tecnológico (WALLERSTEIN, 2005 BLINDER, 2017).

4. En efecto, el sector agroindustrial, minero o petrolero puede ser un asunto de disputa comercial entre un país avanzado y uno que no lo es, en tanto que países avanzados cuentan con un sector agrario importante como es el caso de Estados Unidos o algunos países europeos. Sin embargo, el conflicto llega cuando se trata de un sector estratégico que implica tecnología cuyo desarrollo detentan los países centrales, y solo un puñado de estos.

5."En cuanto a las capacidades materiales, si el ciclo de expansión económica de posguerra se basó en el fuerte

aumento de la producción impulsado por el fordismo, el ciclo expansivo de la globalización se explica por la aparición, desde finales de los años setenta, de un nuevo modelo posfordista transnacional basado en la segmentación, externalización y deslocalización

del proceso productivo. Ese modelo comporta nuevas formas de integración horizontal de la producción en cadenas globales de suministro (global supply chains) protagonizadas por empresas multinacionales, que se tradujo en un crecimiento muy intenso del comercio intra-firma. Ello ha supuesto un amplio desplazamiento de la manufactura y de servicios a países con menores costes de mano de obra, inicialmente en actividad de baja calificación, y después en otras más calificadas. Con ello, desde los años ochenta se produjo un notable aumento de la inversión extranjera directa (IED) de empresas multinacionales. Aunque los países avanzados han retenido las actividades productivas más intensivas en conocimiento, se ha producido un rápido proceso de difusión de tecnología hacia los países emergentes. A ello contribuyó la caída de costes del transporte y las comunicaciones -trasporte multimodal y containers, "Toyotismo" u organización just-in-time de la logística, telefonía celular e Internet-y desde los noventa cambios tecnológicos que permiten la integración digital global de las cadenas de valor" (SANAHUJA, 2017, p. 48). rápido crecimiento y se aplican a distintas ramas de la producción con múltiples propósitos productivos, para uso bélico o comercial. Sin embargo, los sectores estratégicos que nos importan refieren a las Tecnologías de propósito general, puesto que si bien para determinado Estado semiperiférico ${ }^{3}$ puede resultar estratégico el sector primario, este no resulta conflictivo ni compite con las economías centrales ${ }^{4}$. Las Tecnologías de Propósito General tienen una aplicabilidad general, siendo útil o para uso de otras tecnologías y procesos productivos, presentan un gran dinamismo, y allí donde se aplican tienen la capacidad de potenciar innovaciones en distintos sectores de la economía.

Geopolítica y economía

Vivimos en una etapa de transición ${ }^{5}$ a partir de la crisis de económica global de 2008, en la cual se materializan nuevos polos de poder económicos. La gobernanza global también está en estado de dificultad encontrándose la etapa actual de la globalización sin multipolaridad, y con un modelo de transnacionalización productiva que va agotándose en sus límites sociales, políticos y ecológicos. Las potencias emergentes adquieren estatus de potencia económica, aunque bajo la multipolaridad hegemónica de posguerra, liderada por Estados Unidos, pero con problemas de representatividad y legitimidad de los países emergentes (SANAHUJA, 2017).

Pero el cambio profundo y estructural radica en que está en proceso una nueva revolución industrial signada por el agotamiento de la globalización como ciclo económico: valorización y volatilidad financiera, debilidad de inversión y comercio. "Se estaría cerrando un ciclo productivo posfordista [...] ante cambios tecnológicos que alientan dinámicas de re-localización productiva, automatización e integración de las cadenas de valor a través de las plataformas digitales [...]” (SANAHUJA, 2017, p. 45). Estos cambios producen transformaciones sobre el empleo, el bienestar social, los acuerdos distributivos, y la política debido a los efectos sobre la ciudadanía y sus demandas que no pueden ser respondidas por el Estado Nación.

China es la potencia industrial emergente más importante después de Estados Unidos. En el año 2015, los planificadores de política chinos promovieron el desarrollo de industrias avanzadas en ese país. Si bien China se encuentra entre los países manufactureros más importantes globalmente, lejos de hacer políticas públicas de libre mercado, los planificadores buscan desarrollar industrias estratégicas que son aquellas con las que cuentan los países avanzados. Los chinos producen en porcentaje sobre el total mundial, "el $28 \%$ de los automóviles, el $41 \%$ de los barcos, $80 \%$ de las computadoras, el $90 \%$ de los teléfonos celulares, el $60 \%$ de los televisores, el $50 \%$ de las heladeras, el $80 \%$ de los aires acondicionados, $24 \%$ de la energía, y el 50\% del hierro" (EUROPEAN CHAMBER, 2017, p.2). A partir de la crisis financiera global de 2008, "el país emergió como el mayor motor económico mundial” (LÜTHJE, 2014, p. 3).

La estrategia china se basa en una economía que se denomina de conocimiento. Sin embargo, esta economía que implica alto valor agre- 
gado no quiere decir que emplee más conocimiento que en tiempos pretéritos, sino que el conocimiento se ha convertido en un commodity, un bien económico. Ya hacia "fines del Siglo XIX el mundo de los negocios comenzó a reconocer la importancia del conocimiento tecnológico como activo estratégico" (CORIAT; WEINSTEIN, 2011, p. 273). La cuestión central deviene, entonces, en quién es el dueño de este conocimiento: para ello se fue modificando la legislación que reglamentaba la apropiación por parte de los dueños de las compañías. Pero el cambio del neoliberalismo en la década de 1980 y la transición hacia un modelo fordista de producción llevó a nuevas regulaciones como la Bayh-Dole Act en los Estados Unidos, que permitía el patentamiento con fondos aportados por el gobierno federal, lo que antes no era posible, siendo obligatorio que todo desarrollo financiado gubernamentalmente debía ser aplicado en el Estado (CORIAT; WEINSTEIN, 2011). Las consecuencias materiales de esta regulación permitieron a universidades, laboratorios o firmas desarrollar comercialmente una tecnología financiada por el Estado, "un boom espectacular de spin-offs" (CORIAT y WEINSTEIN, 2011, p. 282).

Estos procesos reducen la importancia de las empresas multinacionales y las economías de escala: en los países emergentes en donde se radicaban las multinacionales en la era de la globalización, las ventajas competitivas dejan de serlo puesto que aumentan los salarios orientando las economías hacia el mercado interno. La lógica actual de esta fase posglobalización alienta a situar la producción más cerca de los consumidores, ya sea en los países avanzados, como en los emergentes en donde existe una creciente clase media, relocalizando la producción (SANAHUJA, 2017). Esto quiere decir multinacionales de los países centrales produciendo en el mercado de países semiperiféricos, pero también vendiendo para ellos; también quiere decir empresas de estos últimos países produciendo en y para mercados de países centrales.

La evidencia empírica muestra que la implementación de cambios en el mundo productivo a partir de la robótica y la impresión 3D está generando modificaciones permanentes y spillovers por lo que estamos en una nueva implementación de Tecnologías Estratégicas y de Propósito General. En efecto, este proceso no solo ha disminuido la cantidad de empleos industriales sino que también se ha trasladado a países semiperiféricos mediante el proceso de outsourcing, en los cuales no solo mejora la calidad de los puestos de trabajo con mayor valor agregado, sino que también hace peligrar los puestos de trabajo que no emplean esta tecnología. Algunas compañías como la Hon Hai Precision Industry o Foxconn, que fabrica para las principales marcas de las industrias high-tech de los Estados Unidos invierten activamente en robots y 3D (BOGUE, 2014).

La manzana, y sus ramas chinas

De las empresas no estratégicas a estudiar, la primera en el ranking es Apple. La importancia del éxito comercial de esta empresa se debe a varios factores, entre ellos la gran escala de producción de sus fábricas y las cadenas de suministro de las cuales las más importantes se encuentran en China y varios países de Asia (CHAN, et al., 2013). Pero Microsoft, Google 
(hoy parte de Alphabet), IBM y Amazon son clientes que producen algunas de sus manufacturas tecnológicas con Foxconn en China (CHAN, et al., 2013; THE GUARDIAN, 2012; INTERNATIONAL BUSINESS TIMES, 2012; IBM, 2009; COMPUTER WORLD, 2010). ¿Por qué producen en China? Porque tiene formidables ventajas de producción para manufacturas de bajo costo y de alto valor, que demanda el mercado mundial, y crecientemente, el mercado interno chino (THE ECONOMIST, 2015). China es un mercado creciente para la subcontratación/externalización de la producción mundial (FORBES, 2014).

Foxconn tiene distribuida su producción en distintas partes del territorio chino, en donde produce todo tipo de artefactos tecnológicos para empresas estadounidenses: según Google Earth, esta empresa tiene distintas plantas de producción, dos establecimientos en Chongqing, tres en Kunshan, dos en Shenzhen, uno en Huaian, y uno Yantai.

La producción de estas empresas tecnológicas en China no solo se debe a circunstancias de índole costo/beneficio, sino a que son aparatos tecnológicos de consumo masivo: se producen y consumen en todas partes del mundo allí donde exista un mercado para esos productos. Verdaderamente no importa si más o menos teléfonos inteligentes son vendidos a oficiales de inteligencia o militares de China o cualquier país con alguna rivalidad geoestratégica con los Estados Unidos. Un oficial militar de cualquier país con cierto poder de fuego, así como cualquier ciudadano puede tener una computadora de marca estadounidense y fabricada en China. En principio eso no afecta seguridad nacional alguna. Sí afecta -naturalmente- la performance económica de la empresa y su rentabilidad. Pero Apple, Microsoft, Google Alphabet, IBM o Amazon pueden ser producidos y consumidos en cualquier lugar. Boeing, Lockheed Martin, General Dynamics, Raytheon, Northrop Gruman, no. Son tecnologías estratégicas.

Cuando hablamos de tecnologías comercialmente exitosas y de uso civil, también estas tuvieron un origen en la producción relacionada a la política y producción de Defensa, y un fuerte financiamiento del Estado central. El Estado generó las condiciones para que existiera innovación y desarrollo invirtiendo desde las arcas gubernamentales activamente parte del presupuesto para desarrollar sectores estratégicos. Se generaron así condiciones para el desarrollo, puesto que los capitales privados son siempre aversos al riesgo. La investigación básica la financia el sector público. Cuando llega el momento de comercializar, interviene la inversión privada como fue el caso de Internet, los semiconductores o la industria aeroespacial (MAZZUCATO, 2013), que son, en efecto, todos los casos que tratamos en el presente trabajo. 
Imagen 1 - Instalaciones de Foxconn en China.

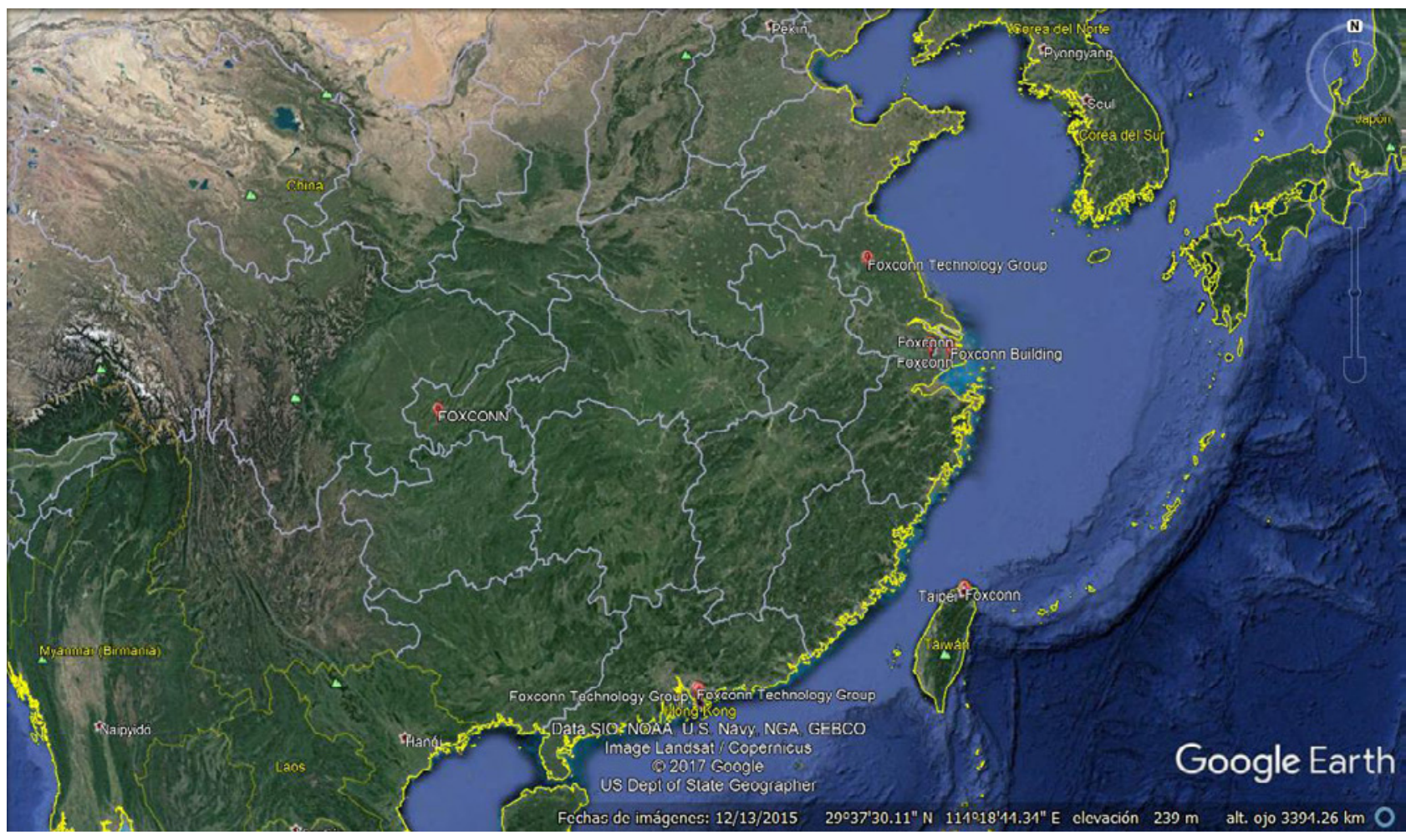

Fuente: Google Earth con edición propia (GOOGLE EARTH, 2017).

Solo hecho en América

Las tecnologías estratégicas no se producen en China, sino en Estados Unidos. Son parte de aquello que se denominó el Complejo Militar-Industrial del cual formaban parte no solo el Estado federal, sino empresarios, y otros actores de poder. Aquí juega fuerte la geopolítica, puesto que están ancladas dentro del marco territorial soberano. Eso no quiere decir que estas compañías hagan su juego solo localmente dentro del marco de la escala nacional. Estas empresas colocan sus productos en países aliados militarmente, como el Reino Unido, Israel, Arabia Saudita y otros (SIPRI FACT SHEET, 2017).

Estamos aquí hablando de corporaciones de gran tamaño y grandes ganancias con influencia económica y política en Estados Unidos y otros países. Sin embargo, las diez compañías vinculadas al consumo masivo de tecnología o del sector Defensa, todas colocan dividendos en guaridas fiscales (CITIZENS TAX JUSTICE, 2016), fugando dinero que se evitan tributar en su país de origen: en ese sentido, estamos discutiendo empresas que no solo influencian a la política militar e internacional, sino que también se comportan como cualquier actor económico que busca maximizar su lucro independientemente de la seguridad financiera del Estado o de los ciudadanos que con sus aportes impositivos, financian la maquinaria bélico-industrial que estos aportan para la consecución de una hegemonía global. Son actores globales, pero las tecnologías estratégicas tienen anclaje productivo local/nacional, mientras que las demás global. 
Los militares de alto nivel en lugares de decisión del Pentágono en Estados Unidos han tenido una gran autonomía de decisión y grandes recursos que han dirigido la política de producción bélica en ese país, imprimiendo su huella en las decisiones de alta política dado que el tipo de industria que se desarrolló se constituyó en los medios para un objetivo de política exterior y de seguridad, pero -paradójicamente- muy influenciada por los propios burócratas de armas. Mientras la potencia vencedora de la Segunda Guerra Mundial tuvo por un breve tiempo el monopolio de las armas nucleares, el gasto bajó abruptamente, sobre todo tras el fin de la contienda en 1945. Pero una vez que la Unión Soviética consiguió la bomba atómica en 1949, esta tendencia se revirtió, y muchos dineros fueron desde las arcas del Estado a Defensa para el incipiente Complejo Militar-Industrial (HOOKS, 1990).

Las empresas estadounidenses analizadas tienen distribuida su producción solo en este país, y en distintas partes del territorio, en donde producen todo tipo de artefactos tecnológicos estratégicos: según Google Earth, estas empresa tiene distintas plantas de producción a lo largo de esta geografía.

Lockheed Martin tiene facilidades en Bethesda (Maryland), Chelmsford (Massachusetts), Palo Alto (California), Grand Prairie (Texas), Richardson (Texas), North Charleston (North Carolina), Edison (New Jersey), Huntsville (Alabama), Colorado Springs (Colorado), Lakeland (Florida). Boeing las tiene en Mesa (Arizona), Arlington (Virginia), Oklahoma City (Oklahoma), Chicago (Illinois), North Charleston (South Carolina), Richardson (Texas), Ridley Park (Pennsylvania), Kent (Washington), Seal Beach (California), Colorado Springs (Colorado). Raytheon tiene sus instalaciones en Forest (Misisipi), Fort Wayne (Indiana), Raleigh (North Carolina), Albuquerque (New Mexico), Lawton (Oklahoma), Goleta (California), Colorado Springs (Colorado), Fort Worth (Texas), El Segundo (California), Herndon (Virginia). Northrop Gruman las tiene en St Augustine (Florida), Atlanta (Georgia), Woodland Hills (California), Irving (Texas), Raleigh (North Carolina), Huntsville (Alabama), Redondo Beach (California), Boulder (Colorado), Baltimore (Maryland), Charlotte (North Carolina). Por último, General Dynamics tiene sus facilidades en Rochelle Park (New Jersey), Riverview (Florida), Hattiesburg (Mississippi), San Diego (California), Sterling Heights (Michigan), Fayetteville (North Carolina), Panama City (Florida), Fairfax (Virginia), Saco (Maine), y Corbin (Kentucky).

Estamos en una era de outsourcing, offshoring, alianzas, asociaciones, redes, y clusters desde que se abandonó la idea de internalizar las funciones tanto hacia arriba como hacia abajo de la cadena de suministro por efecto de la globalización (IAMMARINO; MC CANN, 2013). Pero también de reshoring en la cual las empresas están relocalizando en zonas más próximas la producción debido a distintas razones, especialmente por los costos del transporte, de la mano de obra que ya no es tan barata en otras partes del globo y además por la calificación de la misma, y la proximidad de los mercados (SANAHUJA 2017; FRATOCCHI et al., 2016; FRATOCCHI et al., 2014; TATE, 2014). De esta forma hay razones para creer que los países con capacidad de producción doméstica de tecnología 
avanzada, tendrá mayores capacidades para reabsorber aquellos procesos industriales que vuelven a relocalizarse en su país, mientras que por el contrario, aquellos países que tuvieron durante los años de la globalización un fuerte crecimiento de industrias por el offshoring de los países centrales, y que han comenzado a desarrollar sus propias marcas y diseños -no meramente copiar- tendrán más chances de desarrollar un fuerte entramado de lo que en este trabajo hemos llamado industrias estratégicas. Todo esto dependerá de la geopolítica.

Imagen 2 - Instalaciones de empresas estratégicas en EEUU.

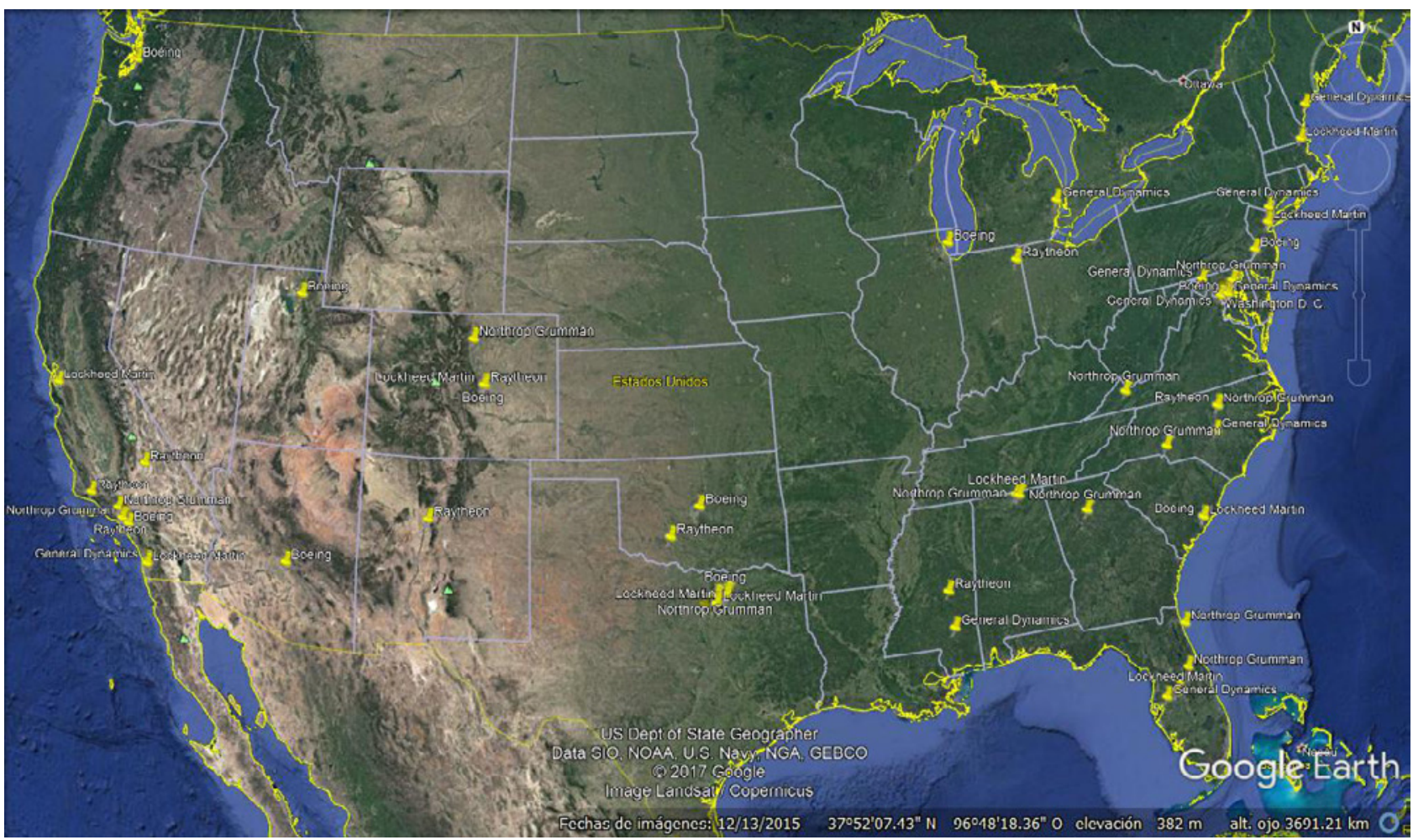

Fuente: Google Earth (GOOGLE EARTH, 2017).

Geopolítica de la tecnología

Parte de la evidencia encontrada es que las empresas que producen tecnologías estratégicas producen dentro de las fronteras nacionales de bandera mientras que las que no lo hacen dentro o fuera, pero su elección se debe a decisiones que obedecen a otro tipo de racionalidad instrumental, vinculada a la eficiencia, no a la seguridad o al poder nacional como en el primer caso. Esto responde a una espacialidad del poder, en este caso, el productivo. El poder político está asociado muy fuertemente al Estado moderno, y este tomado como un actor unitario, "como unidades de soberanía territorial [...], buscando mayor poder fuera de sus fronteras" (AGNEW, 2005, p. 37). De esta manera existe una rígida concepción territorial en la cual las unidades estatales operan por un sistema de estados territoriales: "una concepción más rica del contexto espacial mira al territorio del Estado como una de las tantos encuadres geográficos del poder político" (AGNEW, 2005, p. 39). Así, el poder no es una sumatoria 
de capacidades -en este caso industriales- ni una posesión ejercida por un Estado que se comporta por motivos homologables a las necesidades, pasiones, o moral de poder del individuo.

Gráfico 1 - Ingresos y valor total de la compañia en billones de US\$ - Forbes (2017)

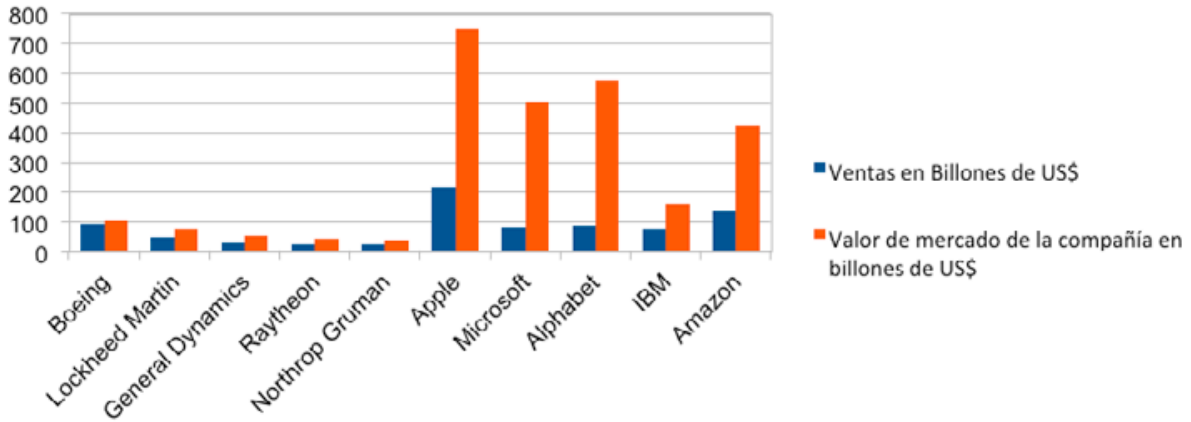

Fuente: elaboración propia en base a Forbes.com

El Estado, por el contrario, es una institución de poder, una más en un complejo entramado de relaciones. Dentro de su propia lógica de funcionamiento, se desenvuelve para conseguir los objetivos de poder de distintos actores que por lo general tienen influencia o poder sobre esta estructura institucional. Esta estructura favorece los proyectos industriales de las empresas en el territorio soberano o fuera de él. No obstante, dentro de las fronteras nacionales se fomentan y desarrollan las industrias de tecnologías estratégicas cuya lógica dual implica alto desarrollo, compra del Estado, uso bélico y poder. Pero esta es una de las tantas lógicas que operan superpuestas: para la observación de la producción offshore, no debe caerse en la trampa territorial, "que tiene muy enraizado el concepto por el cual se asume que la soberanía estatal moderna requiere delimitaciones claras del territorio que encierra” (AGNEW, 2005, p. 41).

La idea del poder de compra estatal no es nueva, y responde a mecanismos de proteccionismo y desarrollo endógeno de capacidades, siendo un mecanismo por el cual el Estado selecciona qué y cómo. En palabras de Portantiero (1989, p. 162) el

\footnotetext{
"financiamiento que la sociedad, [que] vía el gasto público, le otorga al sector privado, asume formas diversas, pero que pueden ser resumidas en cuatro fundamentales: los subsidios directos, las exenciones impositivas, la orientación del poder de compra del Estado y el proteccionismo, como salvataje corporativo de los riesgos de la competencia”.
}

Así, entendemos un instrumento que se da en las economías avanzadas de los países centrales:

En los países avanzados, la relación entre la gran empresa y el Estado parece alcanzar, en términos generales, un grado elevado de articulación; es una interdependencia que $[. .$.$] no esta exenta de conflictos, pero que en lo funda-$ mental parece responder coherentemente con los objetivos de funcionamiento y expansión del sistema en su conjunto. Las empresas buscan por parte del Estado, entre otros elementos, los siguientes: la legitimidad social para la función que ellas desempeñan. Es decir, el Estado debe avalar jurídica y políticamente la actividad productiva de estas grandes empresas. [...] La gran empresa aspira a que la acción económica del Estado, fundamentalmente en lo que se refiere a las políticas de inversión, financia miento, subsidios y precios conformen un contexto económico coherente con sus necesidades y capacidad de expansión [...] [Aquí] pueden mencionarse el poder de compra del Estado como meta de la 
política de acercamiento por parte de las grandes empresas, ya sea cuando se trata de servicios destinados al uso civil o al militar. Ademas del "mercado publico" las empresas requieren la contribución financiera del Estado para el desarrollo de las actividades de investigación y desarrollo, y para la formación de los cuadros técnicos (FAJNZYLVER, 1976, p.29).

En consecuencia asumir que existe una política doméstica o externa es incorrecto dado que el mundo no se delimita por las líneas limítrofes. Sí existe una política exterior, es aquella política que hace un Estado hacia otro Estado, pero está entramada con una serie de otras políticas, de otros actores no estatales, o estatales de otros niveles. En realidad, lo que está sucediendo es un proceso en el cual la geopolítica se está desterritorializando, que es el proceso de la declinación de la soberanía estatal dada la incapacidad de controlar -léase ejercer poder- los flujos comerciales, la información, y las personas (FLINT, 2001).

Aquí se juegan dos lógicas distintas: el cliente. El principal cliente de las primeras compañías no estratégicas son actores privados. La lógica mercantil prima sobre cualquier otra, como oferta y demanda. Opuestamente, las tecnologías estratégicas tienen como cliente al Estado, y prima la lógica de racionalidad estratégica, de poder de compra del Estado, de seguridad nacional. Evidentemente las compañías estratégicas analizadas tienen también líneas de desarrollo civil, pero concretamente nos referimos a los desarrollos de tipo militar.

Aquí la competencia se da en los mecanismos oferentes de licitación en aquellos proveedores con security clearence (autorización de acceso para personas o empresas que cumplen con protocolos de seguridad). En este último caso, es un mercado mucho más regulado y direccionado, en el cual existe una construcción de confianza entre los proveedores de la tecnología y los organismos estatales, para que no se comparta la información de la investigación y desarrollo del producto, ni con empresas capaces de replicar copias de la ingeniería, ni con ningún Estado. La construcción del artefacto se hace mirando el propósito del mismo, y las necesidades tácticas que entre militares, técnicos y burócratas civiles deciden para cumplir con los planes de Defensa Nacional (BIJKER y PINCH, 2013; MACKENZIE, 2012).

La cuestión del poder de compra del Estado y la seguridad es primordial, puesto que refiere a dos procesos relevantes de la geoeconomía. En primer lugar, al incentivo mentado, al ya mencionado direccionamiento del desarrollo tecnológico en base a las políticas de la burocracia estatal. ¿Qué se va a desarrollar? ¿Cuántos recursos disponemos? ¿Cuál es el objetivo que queremos conseguir? Obtener ciertas capacidades tecnológicas -que son producidas en este caso por las cinco empresas estratégicas- es articular y aplicar capacidades para la política de Defensa que tiene aplicaciones concretas en una operación militar, pero también en el liderazgo de la vanguardia de la tecnología en ese segmento. El Estado, al garantizar la compra, asegura no sólo la venta de todas las unidades de la serie de artefactos producidos por la industria, sino que resulta el incentivo final para el desarrollo de artefactos que nadie más compraría por poder destructivo y escala. Evidentemente, también hay competencia en este caso. Los pocos Estados o empresas con capacidades similares buscarán equilibrar el poder de fuego, en un mercado extremadamente regulado y controlado, porque mediante espionaje, ingeniería, o meramente investigación y 
6. China está produciendo tecnologías que pueden ser consideradas estratégicas por el largo proceso de desarrollo e industrialización que tuvo por décadas, también en parte a la llamada ingeniería reversa. Algunas de las empresas estadounidenses que aquí llamamos estratégicas producen en China, y todo indica que esta tendencia seguirá. Sin embargo, no producen la tecnología militar cuya regulación está al orden del día dada la competencia en este campo por parte de ambos países, puesto que China se encamina también a obtener el status de potencia militar desarrollo, buscarán equiparar, por lo que los incentivos de las empresas estratégicas y de los Estados para financiar nuevos desarrollos no acaba.

El desarrollo tecnológico estatal se relaciona con la inserción en un orden internacional, la capacidad de gestionar la diplomacia, las regulaciones internacionales, y los negocios a nivel global. En palabras de Ha-Joon Chang, "el desarrollo económico consiste en adquirir y dominar tecnologías avanzadas" (2008, p. 81). De acuerdo a Schoonmaker (2002), el discurso de la globalización oculta conflictos políticos y económicos. colocar mercancías con menor valor agregado en el mercado mundial, implica para los países de la periferia la continuidad de su situación.

Pero algunos países no centrales han tomado rumbos que acarrean el desarrollo de determinadas industrias relacionadas con tecnologías avanzadas, a costa de guerras comerciales, sanciones, competencia desleal, presiones diplomáticas formales e informales y fuerte intervención por parte de las empresas líderes con base en los países centrales. Este mercado deja poco lugar a la competencia, deja lugar a una fuerte regulación, mantiene el control de determinadas industrias avanzadas y de uso dual por parte de pocos actores, y limita la competencia solo a ellos. Por eso se puede hablar de un Orden Mundial Tecnológico (BLINDER, 2017). La producción de mercancías de tecnología no estratégica pueden producirse fuera de las fronteras nacionales y responde a la lógica de la globalización ${ }^{6}$.

Reflexiones finales

Pensar la locación de la producción, en clave de importancia geopolítica, de las principales compañías de tecnologías de telecomunicaciones, las productoras de tecnologías satelitales y las principales productoras de teléfonos celulares, computadoras, internet y otros artefactos de la computación nos ancla necesariamente al territorio y nos obliga a pensar en él. Analizando la geopolítica de las industrias estratégicas y las que no lo son, a partir del análisis de los casos de las cinco principales compañías de tecnología encontramos factores determinantes al momento de caracterizar cuáles son estratégicas y cuáles no, dónde pondrán estas sus plantas de producción, y las razones instrumentalizadas del por qué la elección geográfica de la planta de producción.

Las lógicas geopolíticas variarán de acuerdo al nicho tecnológico a fabricar: la elección para el desarrollo de las tecnologías estratégicas estará completamente mediatizada por argumentos de seguridad nacional, y de obtención de beneficios a través de la compra del las burocracias estatales de la Defensa y la Seguridad, así como de la política exterior hacia otros Estados que la diplomacia considera aliados. Quizás podría encontrarse mano de obra especializada en otros países, pero aquí la rentabilidad del negocio no es el argumento final. Tiene sentido: aviones de combate, drones, sistemas de armas aeroespaciales no se producen en masa. Muy por el contrario, las no estratégicas sí son producidas masivamente, y la fórmula costo-beneficio se presenta como la más importante, lo cual decide la localización de las facilidades de producción.

En suma, los motivos para producir en el propio territorio depende más que nada de quién es el cliente, y cuál es su propósito, siendo el issue 
militar un argumento de peso a la hora de pensar integralmente la producción con la política de Defensa de y Seguridad Nacional. El volumen del mercado, sin embargo, no es determinante para producir en el país de bandera, sino que por lo menos en las cinco tecnologías no estratégicas aquí trabajadas, tienden a trasladarse fuera de las fronteras por cuestiones de ventajas comparativas en la economía de la producción. Estamos hablando de compañías que ganan billones de dólares. Por el contrario, el tipo de mercado si juega un papel crucial como se explicó para el otro grupo analizado, pues es el Estado quien es el comprador por motivos de alta política en el cual deciden invertir en tecnologías estratégicas. Aquí son negocios billonarios también, pero mucho menor en cantidad.

Los cambios geoeconómicos de la posglobalización están modificando las pautas de producción de las industrias, llevando a producir allí donde existe abaratamiento y competitividad en materia impositiva, de transporte, proximidad, cualificación de los operarios, y disponibilidad técnica. Toda este proceso que es de consumo masivo y que por ingresos, de acuerdo a Forbes están muchísimo mejor posicionadas en cuanto a beneficios obtenidos que las industrias estratégicas, representa la mayor parte de la dinámica geopolítica a escala global. Solo rompemos con la desterritorialización cuando el poder de compra es del Estado, cuando es la guerra o la superioridad estratégica aquella que guía las decisiones.

Resta reflexionar acerca del resto de la lista que prosigue a la selección de cinco casos. ¿El fenómeno es similar, se repite el patrón? ¿Hay excepciones? Este es objeto de futuros estudios en que se requiere rastrear el listado completo. Las afirmaciones herméticas esgrimidas al principio acerca de que tanto es arriba como abajo no aplica en efecto a la política tecnológica. Dominar todo el rango necesario de la tecnología que va arriba, en altura, para iluminar con su señal a Tierra, como también tener la infraestructura terrestre, son condiciones indispensables para tener el dominio de todo el espectro. Mas producir las tecnologías de abajo, de Tierra, como los software y hardware necesario para las comunicaciones, si bien son un mercado codiciable y billonario, no son un negocio estratégico: quizás sí, estrictamente, si hablamos del significado del tamaño del negocio para el capitalista y su Estado de procedencia.

Opuestamente, las tecnologías de arriba, como las satelitales si lo son. Las tecnologías estratégicas parecen por su posición cumplir con la etimología de la palabra estrategia, el arte de dirigir ejércitos: arte que se ejercía en el campo de batalla en una posición más elevada del terreno, para ver todo desde las alturas. Colocar un satélite en órbita es un proceso complejo que unos pocos gobiernos y contratistas pueden lograr. Fabricarlo, también. Lo mismo sucede con otras industrias aeroespaciales. Eso explica que la producción se encuentre asegurada, allí donde las capacidades de ejercicio de soberanía son plausibles, y las fuerzas del Estado tienen un mayor nivel de control.

Bibliografía

AGNEW, John. Hegemony. The new shape of global power. Philadelphia: Temple University Press, 2005.

BIJKER, W. y PINCH, T.,. La construcción social de hechos y artefactos: o acerca de cómo la sociología de la ciencia y la sociología de la tecnología pueden beneficiarse mutuamente. En: 
THOMAS, H. y BUCH, A. (Coords.). Actos, actores y artefactos. Sociología de la Tecnología. Quilmes: Universidad Nacional de Quilmes, p. 19-62,2013.

BLINDER, Daniel. Orden Mundial y tecnología. Análisis institucional desde la perspectiva geopolítica en la semiperiferia: la tecnología espacial y de misiles en Argentina y Brasil. Revista Geopolítica(s), Universidad Complutense: Madrid. v.8, n. 2, p. 177-202,2017.

BOGUE, Robert. What future for humans in assembly?. Assembly Automation, v. 34, n. 4, p. $305-309,2014$

CHAN, Jenny; PUN, Ngai; SELDEN, Mark. The politics of global production: Apple, Foxconn and China's new working class. New Technology, v. 28, n. 2,. p. 100-115, 2013.

CHASE-DUNN, Christopher; REIFER, Thomas. US Hegemony and Biotechnology: The Geopolitics of New Lead Technology. Working paper $n^{\circ}$ 9. Institute for Research on World Systems, Universidad de California. 2002. Disponible en: $<$ http://irows.ucr.edu/papers/irows9/ irows9.htm> Consultado: 20 Sep. 2016.

CHANG, Ha-Joon. Bad Samaritans. The Myth of Free Trade and the Secret History of Capitalism. New York: Bloomsbury, 2008.

CITIZENS FOR TAX JUSTICE. "Fortune 500 Companies Hold a Record \$2.4 Trillion Offshore”. 2016. disponible en: < http://ctj.org/ctjreports/2016/03/fortune 500 companies hold a record 24 trillion offshore.php\#.WcQqZdFrzIU>Consultado: 21 de Sep. 2016.

COMPUTER WORLD. "Kindle screen maker will increase capacity to meet demand". $28 \mathrm{Jul}$. 2010. Disponible en: < https://www.computerworld.com/article/2519700/smartphones/kindle-screen-maker-will-increase-capacity-to-meet-demand.html> Consultado: 30 Agosto 2017.

CORIAT, Benjamin; WEINSTEIN, Oliver. Patent regimes, firms and the commodification of knowledge. Socio-economic Review. v.10., p.267-292. 2011.

CUPITT, Richard. Reluctant Champions. U.S. Presidential Policy and Strategic Export Controls. New York. Routledge. 2000.

EUROPEAN CHAMBER. China Manufacturing 2025. Putting Industrial Policy Ahead of Market Forces. European Union Chamber of Commerce in China. p. 1-64. Disponible en: <www. europeanchamber.com.cn> Consultado: 30 Ago. 2017.

FAJNZYLBER, Fernando. Oligopolio, empresas trasnacionales y estilos de desarrollo. El Trimestre Económico. v.43., $N^{\circ}$ n. 171(3)., p.625-656. 1976.

FLINT, Colin. The geopolitics of laughter and forgetting: A worldsystems interpretation of the postmodern geopolitical condition. Geopolitics. v.6, n. 3., p. 1-16. 2001.

FORBES. "Manufacturing In China Can Give Your Business The Competitive Advantage". 07 Feb. 2014 Disponible en: < https://www.forbes.com/sites/allbusiness/2014/02/07/manufacturing-in-china-can-give-your-business-the-competitive-advantage/\#33a9af352774> Consultado: 18 Sep. 2017.

FORBES. The World's Largest Public Companies. https://www.forbes.com/global2000/list/ Consultado: 11 Septiembre 2017.

FRATOCCHI, L.; ANCARANI, A.; BARBIERI, P.;DI MAURO, C.; NASSIMBENI, G.; SARTOR, M.; VIGNOLI, M.; ZANONI, A. Motivations of manufacturing reshoring: an interpretative framework. International Journal of Physical Distribution \& Logistics Management. v.46 n. 2,. p. 98-127. 2016.

FRATOCCHI L.; DI MAURO C.; BARBIERI P.; NASSIMBENI G.; ZANONI A. When manufacturing moves back: Concepts and questions. Journal of Purchasing \& Supply Management. v. 20., p 54-59. 2014.

GERSHWIN, Lawrence. Foreword. En DAVIS, Z., LEHMAN, R., NACHT, M., et. al. Strategic Latency and World Politics: HowTechnology is Changing Our Concepts of Security. Center for Global Security Research at Smashwords. Lawrence Livermore National Laboratory: Livermore CA. 2014.

GOOGLE EARTH. Consultado: 02 Oct 2017.

HOOKS, Gregory. The Rise of the Pentagon and U.S. State Building: The Defense Program as Industrial Policy. American Journal of Sociology. v. 96, n. 2., p.358-404. 1990.

HURTADO, Diego; LUGONES, Manuel; SURTAYEVA, Sofya. Tecnologías de propósito general y políticas tecnológicas en la semiperiferia: el caso de la nanotecnología en la Argentina. Revista CTS, n 34, v. 12, p. 65-93. Feb. 2017. Disponível em: https://www.redalyc.org/ html/924/92452927003/ 
IAMMARINO, Simona; MAC CANN. Multinationals and Economic Geography Location, Technology and Innovation. Massachusetts. Elgar. 2013.

IBM. "Foxconn Teams With IBM to Fight Asia Pacific Region's Carbon Emissions". 04 Mar. 2009. Disponible en: $<$ https://www-03.ibm.com/press/us/en/pressrelease/26836.wss $>$ Consultado: 11 Septiembre 2017.

INTERNATIONAL BUSINESS TIMES. "Chinese Contractors: Foxconn's Underage Worker Use Affects Sony, Google, Apple, Amazon, Nokia”. 17 Oct. 2012. Disponible en: < $\underline{\text { http://www.ib- }}$ times.com/chinese-contractors-foxconns-underage-worker-use-affects-sony-google-apple-amazon-nokia-847987> Consultado: 11 Sept 2017.

LEHMAN, Ronald. Unclear and Present Danger: The Strategic Implications of Latent, DualUse Science and Technology. En: DAVIS, Z., LEHMAN, R., NACHT, M., et. al. Strategic Latency and World Politics: HowTechnology is Changing Our Concepts of Security. Center for Global Security Research at Smashwords. Lawrence Livermore National Laboratory: Livermore CA. 2014.

LÜTHJE, Boy. Why no fordism in China? Regimes of Accumulat ion and Regimes of Product ion in Chinese Manufactur ing Industries. Institut für Sozialforschung. Working Paper $\mathrm{N}^{\circ} \mathrm{n} .3$. Goethe Universität: Franfurt. 2014. Disponible en: $<$ http://www.ifs.uni-frankfurt.de/wp-content/uploads/IfS-WP3-Luethje-Why-No-Fordism-in-China1.pdf $>$ Consultado: 18 Sep. 2017.

MACKENZIE, Donald. "Missile Accuracy: A Case Study in the Social Processes of Technological Change”. En: BIJKER, Wiebe; HUGHES, Thomas; PINCH, Trevor, et al. The Social Construction of Technological Systems: New Directions in the Sociology and History of Technology. MIT University Press: Massachusetts. 2012.

MAZZUCATO, Mariana. The entrepreneurial state: debunking public vs. private sector myths. London Anthem Press. 2013.

MICHALSKI, Wolfgang. Support Policies for Strategic Industries: An Introduction to the Main Issues. p. 7-14. In: OECD. Strategic Industries in Global Economies. Policy Issues for the 1990s. Paris. OECD International Futures Programme. 1991.

NSDD 189 / NATIONAL SECURITY DECITION DIRECTIVE 189. National Policy on Transfer of Scientific, Technical and Engineering Information. National Archives Catalog. En línea: $<$ https://catalog.archives.gov/id/6879779> [Consultado el 13 de Junio de 2018].

PORTANTIERO, Juan Carlos. La múltiple transformación del Estado latinoamericano. Nueva Sociedad, $n^{\circ}$ 104. p.158-168. 1989.

RUTTAN, Vernon. Is War Necessary for Economic Growth? Military Procurement and Technology Development. Oxford: Oxford University Press. 2006.

SANAHUJA, José. Posglobalización y ascenso de la extrema derecha: crisis de hegemonía y riesgos sistémicos. En: Seguridad Internacional y Democracia: guerras, militarización y fronteras. Anuario CEIPAZ 2016-2017. p. 41-77. 2017. Disponible en: < http://www.ceipaz.org/images/ contenido/ANUARIOCOMPLETO\%202017.pdf $>$ Consultado: 30 Ago. 2017.

SCHOONMAKER, Sara. High-Tech Trade Wars. U.S. - Brazilian conflicts in the global economy. Pittsburgh: Pittsburgh University Press. 2002.

SIPRI FACT SHEET. "Trends in International Arms Transfers, 2016”. Stockholm:

Stockholm International Peace Research Institute (SIPRI). 2017. Disponible en: $<\underline{\text { https: } / / \text { www. }}$ sipri.org/sites/default/files/Trends-in-international-arms-transfers-2016.pdf $>$ Consultado: 21 Sep. 2017.

TATE, Wendy. Offshoring and reshoring: U.S. insights and research challenges. Journal of Purchasing \& Supply Management. v. 20. , p. 66-68. 2014.

THE ECONOMIST. "Made in China? Asia’s dominance in manufacturing will endure. That will make development harder for others". 12 Mar. 2015. Dispoible en: $<$ https://www.economist.com/news/leaders/21646204-asias-dominance-manufacturing-will-endure-will-make-development-harder-others-made $>$ Consultado: 18 Sep. 2017.

THE GUARDIAN. "Xbox assembly workers in China threaten mass suicide over jobs dispute”. 12 Ene. 2012. Disponible en: < https://www.theguardian.com/world/2012/jan/12/xbox-assembly-workers-threaten-mass-suicide $>$ Consultado: 11 Sep. 2017.

WALLERSTEIN, Immanuel. El moderno sistema mundial. Tomo I. Méjico: Siglo XXI. 2005. 